\title{
On the Baker-Campbell-Hausdorff Theorem: non-convergence and prolongation issues
}

\author{
Stefano Biagi ${ }^{\mathrm{a}}$, Andrea Bonfiglioli ${ }^{\mathrm{b}}$ and Marco Matone ${ }^{\mathrm{c}}$ \\ ${ }^{a}$ Dipartimento di Ingegneria Industriale e Scienze Matematiche, Università Politecnica delle \\ Marche, via Brecce Bianche, 12, I-60131 Ancona, Italy; \\ ${ }^{\mathrm{b}}$ Dipartimento di Matematica, Università di Bologna, Piazza Porta San Donato, 5, I-40126 \\ Bologna, Italy; \\ ${ }^{\mathrm{c}}$ Dipartimento di Fisica e Astronomia "G. Galilei" and Istituto Nazionale di Fisica Nucleare, \\ Università di Padova, via Marzolo, 8, I-35131, Padova, Italy.
}

\begin{abstract}
ARTICLE HISTORY
Compiled October 15, 2018

ABSTRACT

We investigate some topics related to the celebrated Baker-Campbell-Hausdorff Theorem: a non-convergence result and prolongation issues. Given a Banach algebra $\mathcal{A}$ with identity $I$, and given $X, Y \in \mathcal{A}$, we study the relationship of different issues: the convergence of the BCH series $\sum_{n} Z_{n}(X, Y)$, the existence of a logarithm of $e^{X} e^{Y}$, and the convergence of the Mercator-type series $\sum_{n}(-1)^{n+1}\left(e^{X} e^{Y}-I\right)^{n} / n$ which provides a selected logarithm of $e^{X} e^{Y}$. We fix general results and, by suitable matrix counterexamples, we show that various pathologies can occur, among which we provide a non-convergence result for the $\mathrm{BCH}$ series. This problem is related to some recent results, of interest in physics, on closed formulas for the $\mathrm{BCH}$ series: while the sum of the $\mathrm{BCH}$ series presents several non-convergence issues, these closed formulas can provide a prolongation for the $\mathrm{BCH}$ series when it is not convergent. On the other hand, we show by suitable counterexamples that an analytic prolongation of the $\mathrm{BCH}$ series can be singular even if the $\mathrm{BCH}$ series itself is convergent.
\end{abstract}

\section{KEYWORDS}

Baker-Campbell-Hausdorff Theorem; Matrix algebras; Convergence of the BCH series; Prolongation of the BCH series; Analytic prolongation; Logarithms.

\section{AMS CLASSIFICATION}

Primary: 15A16; 15B99; 40A30. Secondary: 34A25.

\section{Introduction and motivations}

The well-known theorem bearing the names of Baker, Campbell and Hausdorff (BCH, in the sequel) has pivotal applications both in mathematics and in physics: for instance, in the structure theory of Lie algebras and Lie groups (both finite- and infinitedimensional), in group theory, in the analysis of linear PDEs, in the theory of ODEs, in control theory, in numerical analysis (particularly in geometric integration), in operator theory, in quantum and statistical mechanics, in physical chemistry, in statistical 
physics and in quantum field theories. See [1] or the recent monograph [11] for a list of related references.

In very recent years, particularly significant in physics has been the derivation of closed formulas for the BCH series $Z(x, y):=\sum_{n} Z_{n}(X, Y)$, when $X$ and $Y$ are operators satisfying specific commutator relations. Such a progress originated in the paper [43] by Van-Brunt and Visser, and it was soon realized that closed BCH formulas admit relevant extensions by introducing simple algorithms, see [26]. In [27] it has been shown that there are 13 types of commutator algebras admitting such closed forms for the BCH formula. Subsequently, closed BCH formulas for the generators of semisimple complex Lie algebras were derived in [28], where an iterative algorithm generalizing the one in [26] was also introduced.

The above results have been applied in covariantizing the generators of the conformal transformations, in providing explicit expressions of the unitary representations of the fundamental group of Riemann surfaces, in the context of conformal field theories, see [29]. Furthermore, the algorithm in [26] was applied in investigating the zeroenergy states in conformal field theory with sine-square deformation, see [41]. Closed $\mathrm{BCH}$ formulas have been found for the contact Heisenberg algebra, see [14]. Related investigations also concern the recent papers [21,23] on the Zassenhaus formula.

Typically, closed $\mathrm{BCH}$ formulas can be derived by a formal manipulation of the BCH series $Z(x, y)$, often expressed as the logarithm $\ln \left(e^{X} e^{Y}\right)$, or via certain integral representations for the sum of the series. In general, $Z(X, Y)$ coincides with $\ln \left(e^{X} e^{Y}\right)$ only for small norms of $X$ and $Y$ (see Proposition 2.2). Since the power expansion of the exponential is everywhere convergent, it follows that a closed expression, say $L(X, Y)$, for the sum of the series $\sum_{n}(-1)^{n+1}\left(e^{X} e^{Y}-I\right)^{n} / n$ (giving one selected logarithm of $e^{X} e^{Y}$ ), which turns out to be meaningful (and analytic) in a wider region than the set of convergence of that series, will fulfill the identity $e^{L(X, Y)}=e^{X} e^{Y}$ by analytic continuation. The latter identity is very often what physicists look for when dealing with BCH. Equivalently put, closed formulas for the $\mathrm{BCH}$ series obtained in the above way should be referred to as prolongations of the sum of the series $Z(X, Y)$, when the latter is not convergent, which is often the case. Unfortunately, as we will show, the existence and the actual values of $Z(X, Y)$, of $L(X, Y)$, or of their prolongations can be very differently behaved.

Since the coefficients $Z_{n}(X, Y)$ of the $\mathrm{BCH}$ series are well posed in any Lie algebra, the problem of the convergence of the $\mathrm{BCH}$ series $\sum_{n=1}^{\infty} Z_{n}(x, y)$ is meaningful in any Lie algebra equipped with a metric, e.g., in finite-dimensional Lie algebras or in Banach-Lie algebras 1 The study of the convergence domain of the $\mathrm{BCH}$ series has a very long history, tracing back to Hausdorff [22, Section 4], and the determination of the optimal domain of convergence is still an open and trying problem. See, e.g., [4, 7, 9, 13, 15, 18, 30,31,35, 36, 38, 40, 42, 45]. Related references to the convergence domain of the $\mathrm{BCH}$ series focused on its continuous counterpart (of great importance in the applications), the so-called Magnus series, can be found e.g., in [8,32 34].

We do not contribute to the study of the optimality of the $\mathrm{BCH}$ convergence set, which is a highly nontrivial problem, as shown e.g., in [7, 16,33]. Instead, with the use of selected counterexamples, we limit ourselves to showing that the $\mathrm{BCH}$ and logarithmic series $Z(X, Y), L(X, Y)$ can be very differently behaved, as far as convergence/divergence are concerned (see Section 44). The problem of the prolongability of $Z(X, Y)$ (and whether or not this prolongation gives information on the convergence

${ }^{1}$ By a Banach-Lie algebra we mean a Banach space $\mathcal{A}$ (over $\mathbb{R}$ or $\mathbb{C}$ ) endowed with a Lie algebra structure such that $\mathcal{A} \times \mathcal{A} \ni(x, y) \mapsto[x, y] \in \mathcal{A}$ is continuous. 
of $Z(X, Y))$ is also studied here, highlighting some unexpected pathologies.

We would like to highlight some physics applications: there is a basic reason why the convergence issue of the prolongation of the $\mathrm{BCH}$ series is a central question, not only by a purely technical point of view, but also of considerable mathematical and physical interest. It is known that the $\mathrm{BCH}$ Theorem, in its various forms, plays a key role in quantum mechanics, quantum field theories, including their path integral formulation, and statistical physics. Main examples concern the Trotter product formula [39]

$$
\exp (A+B)=\lim _{n \rightarrow \infty}(\exp (A / n) \exp (B / n))^{n}
$$

the Magnus expansion, and its generalizations [2]. To understand this, recall that, for example, the transition amplitude $\left\langle q^{\prime}, t^{\prime} \mid q, t\right\rangle$ that leads to the Dirac-Feynman path integral has the form

$$
\left\langle q^{\prime}, t^{\prime} \mid q, t\right\rangle=e^{-\frac{i}{\hbar} H\left(t^{\prime}-t\right)} \delta\left(q^{\prime}-q\right),
$$

where $H=-\frac{\hbar^{2}}{2 m} \Delta+V(q)$ is the Hamiltonian, $\Delta$ the Laplace-Beltrami operator and $V(q)$ the potential. A similar expression concerns the extension to quantum field theory, where now the Dirac tempered distribution is replaced by the functional Dirac distribution. In most theories, the path integral formulation is treated as a power expansion in the coupling constants. An outstanding problem is that such expansions yield divergent asymptotic series. In recent years a new approach, based on Écalle resurgence theory [19]2, has been developed [3,44]. The main idea is to use transseries expansions, which are faithful and unambiguous representations of observables. In such a construction, the analytic continuation plays the fundamental role. On the other hand, as illustrated by (11), it is clear that the problem of analytic continuation translates into a problem of analytic continuation of the $\mathrm{BCH}$ formula.

Finally, we describe the plan of the paper.

In Section 2 we introduce the precise notation used in the paper along with the statements of the main results. We show that the convergence of $Z(X, Y)$ is totally independent of the existence of $\ln \left(e^{X} e^{Y}\right)$, and even when both $Z(X, Y)$ and $\ln \left(e^{X} e^{Y}\right)$ exist, their values can actually be different. More generally, given a Banach algebra $\mathcal{A}$ with identity $I$, and given $X, Y \in \mathcal{A}$, we study three different issues: the convergence of the $\mathrm{BCH}$ series, the existence of a logarithm of $e^{X} e^{Y}$, and the convergence of the Mercator-type series $\sum_{n} \frac{(-1)^{n+1}}{n}\left(e^{X} e^{Y}-I\right)^{n}$ which provides a selected logarithm of $e^{X} e^{Y}$. We fix general results and, by suitable matrix algebra counterexamples, we show that various pathologies can occur; we provide our main non-convergence result for the $\mathrm{BCH}$ series, for the proof of which a simple Lie-algebraic argument is used.

In Section 3 we give the proofs of the results of Section 2 ,

In Section 4 we exhibit some subtle pathologies which can intervene in the problem of the prolongation of the $\mathrm{BCH}$ series. For instance, we show examples where $Z(X, Y)$ can be analytically prolonged to a function $P(X, Y)$ but:

(1) $P(X, Y)$ has a singularity at $\left(X_{0}, Y_{0}\right)$ but $Z\left(X_{0}, Y_{0}\right)$ converges (Example 2.6);

(2) $P(X, Y)$ is everywhere defined on $\mathfrak{g} \times \mathfrak{g}$ (where $\mathfrak{g}$ is a suitable matrix Lie algebra), whereas $Z(X, Y)$ is somewhere non-convergent (Example 2.7).

\footnotetext{
${ }^{2}$ See 24] for a recent introduction to the related mould calculus applied to the BCH formula.
} 
The example in (2) above is obtained by combining an abstract result in [20], together with a class of examples of Lie algebras of vector fields contained in [12]. The problem of the convergence of $Z(X, Y)$ in Lie algebras of vector fields is of independent interest in the analysis of Hörmander operators (see e.g., [5, 6, 10,12]), and we shall return to it in a future investigation.

\section{Notations and main results}

In its most basic algebraic form, the $\mathrm{BCH}$ Theorem ensures that, in the associative algebra $\mathbb{K}\langle\langle x, y\rangle\rangle$ of the formal power series in two non-commuting indeterminates $x$ and $y$ over a field $\mathbb{K}$ of characteristic zero, one has $e^{x} e^{y}=e^{Z(x, y)}$, where $Z(x, y)$ can be expressed as a series of Lie polynomials

$$
Z(x, y)=x+y+\frac{1}{2}[x, y]+\frac{1}{12}([[x, y], y]+[[y, x], x])-\frac{1}{24}[x,[y,[x, y]]]+\cdots .
$$

To be precise, in the sequel we consider the series $\sum_{n=1}^{\infty} Z_{n}(x, y)$ that can be obtained from $Z(x, y)$ by grouping together the Lie polynomials of degree $n$ in $x$ and $y$, i.e.,

$$
Z_{1}(x, y):=x+y, \quad Z_{2}(x, y):=\frac{1}{2}[x, y], \quad Z_{3}(x, y):=\frac{1}{12}([[x, y], y]+[[y, x], x]), \quad \text { etc. }
$$

This is the so-called homogeneous (presentation of the) $\mathrm{BCH}$ series. Throughout, when a $\mathrm{BCH}$ series is concerned, we always tacitly understand the homogeneous one.

More explicitly, once it is known that $Z(x, y)$ is a Lie series, the $Z_{n}$ 's can be explicitly written, via the Dynkin-Specht-Wever Lemma (as in [11, Sec.3.3.2]), under the following well-know (Dynkin) presentation 3

$$
\begin{aligned}
& Z_{n}(x, y):= \\
& \quad \frac{1}{n} \sum_{k=1}^{n} \frac{(-1)^{k+1}}{k} \sum_{\substack{\left(i_{1}, j_{1}\right), \ldots,\left(i_{k}, j_{k}\right) \neq(0,0) \\
i_{1}+j_{1}+\cdots+i_{k}+j_{k}=n}} \frac{(\operatorname{ad} x)^{i_{1}}(\operatorname{ad} y)^{j_{1}} \cdots(\operatorname{ad} x)^{i_{k}}(\operatorname{ad} y)^{j_{k}-1}(y)}{i_{1} ! j_{1} ! \cdots i_{k} ! j_{k} !} .
\end{aligned}
$$

The convergence of the $\mathrm{BCH}$ series $\sum_{n=1}^{\infty} Z_{n}(x, y)$ in the usua 4 metric topology of $\mathbb{K}\langle\langle x, y\rangle\rangle$ is a trivial consequence of the increasing degrees of the $Z_{n}$ 's.

As this will be relevant throughout the paper, we review that (in the algebraic setting of $\mathbb{K}\langle\langle x, y\rangle\rangle)$ the series $Z(x, y)$ is uniquely given by $\ln \left(e^{x} e^{y}\right)$, where

$$
\ln (W)=\sum_{k=1}^{\infty} \frac{(-1)^{k+1}}{k}(W-I)^{k},
$$

for any formal power series $W \in \mathbb{K}\langle\langle x, y\rangle\rangle$ whose zero-degree term is equal to the identity $I$ of $\mathbb{K}$. Since the power series $\sum_{k=1}^{\infty}(-1)^{k+1} z^{k} / k$ is usually called the 'Mercator series', in order to avoid ambiguities in situations where other logarithms can be meaningful (as in $\mathbb{C}$ or in matrix algebras), we introduce once and for all a selected notation for

\footnotetext{
${ }^{3}$ When $j_{k}=0$ (thus $i_{k} \neq 0$ ) the associated summand in (2) is understood to end with $(\operatorname{ad} x)^{i_{k}-1}(x)$.

${ }^{4}$ See e.g., [11, Section 2.3.3].
} 
what we shall mean by $\ln \left(e^{x} e^{y}\right)$ in more general settings:

$$
\begin{aligned}
& L(x, y):=\sum_{n} L_{n}(x, y), \\
& \text { where } L_{n}(x, y):=\frac{(-1)^{n+1}}{n}\left(e^{x} e^{y}-I\right)^{n} \text { for any } n \in \mathbb{N} .
\end{aligned}
$$

With a little abuse, we say that $\sum_{n} L_{n}(x, y)$ is the Mercator series (a shorthand of 'Mercator series for $\left.\ln \left(e^{x} e^{y}\right)^{\prime}\right)$; we also say that its sum $L(x, y)$ is the Mercator logarithm of $e^{x} e^{y}$, and (when there is no risk of confusion), we may write $\ln \left(e^{x} e^{y}\right)$ in place of $L(x, y)$. The following identity

$$
Z(x, y)=L(x, y) \quad\left(\text { also written as } Z(x, y)=\ln \left(e^{x} e^{y}\right)\right)
$$

is clearly equivalent to $e^{Z(x, y)}=e^{x} e^{y}$, another identity in the formal-power-series setting of $\mathbb{K}\langle\langle x, y\rangle\rangle$ (this equivalence being not always true in other settings).

All these facts are so well established in the $\mathrm{BCH}$ folklore that one may forget that the following four issues, though simple to solve in the formal-power-series setting, may be highly non-trivial if one is working outside $\mathbb{K}\langle\langle x, y\rangle\rangle$ :

(I) the convergence of the Mercator series $\sum_{n=1}^{\infty}(-1)^{n+1}\left(e^{x} e^{y}-I\right)^{n} / n$;

(II) the convergence of the $\mathrm{BCH}$ series $\sum_{n=1}^{\infty} Z_{n}(x, y)$;

(III) the identity $e^{Z(x, y)}=e^{x} e^{y}$, or (generally) the existence of a logarithm of $e^{x} e^{y}$;

(IV) the equality of the sums $L(x, y)$ and $Z(x, y)$ of the series in (I) and (II).

Apart from the algebraic framework of $\mathbb{K}\langle\langle x, y\rangle\rangle$, another kettle of fish is the validity of these four issues when $x$ and $y$ belong to more specific topological spaces, as in the case of matrix algebras. One of the aims of this paper is to study these problems in a wide framework, that of the Banach algebras: we fix some positive general results, and (with the use of selected counterexamples), we show that problems (I)-to-(IV) can be very differently behaved. Indeed, we shall see that many pathological facts do occur even in the simple case of matrix algebras: for example, the $\mathrm{BCH}$ series may converge, whereas the Mercator series may not; or viceversa; or they can be both convergent but with different sums; or they can be both non-convergent, but $e^{x} e^{y}$ may yet admit a logarithm, i.e., some solution $V$ of $e^{V}=e^{x} e^{y}$.

As a result, this will point out some inaccuracies, sometimes appearing in the literature, due to a formal application of the BCH Theorem. Indeed, any formal manipulation (resemblant to what is allowed in $\mathbb{K}\langle\langle x, y\rangle\rangle$ with its very simple topology) of the $\mathrm{BCH}$ series $Z(x, y)$ or of its companion Mercator series $\ln \left(e^{x} e^{y}\right)$ will invariably lose track of all the mentioned pathologies, especially in convergence issues.

In view of the applications, the physics community has paid much attention to the convergence of the $\mathrm{BCH}$ series, as already described. However, some ambiguity occasionally arises from a formal manipulation of the $\mathrm{BCH}$ series. Indeed, when dealing with the $\mathrm{BCH}$ Theorem in physics applications, one often meets with the identity

$$
\ln \left(e^{x} e^{y}\right)=x+y+\frac{1}{2}[x, y]+\frac{1}{12}([[x, y], y]+[[y, x], x])+\cdots .
$$

Unfortunately, while (66) is certainly true in the formal-series setting of $\mathbb{K}\langle\langle x, y\rangle\rangle$, and it is true in any Banach algebra provided that $x$ and $y$ are sufficiently close to zero, it can be dramatically false otherwise. Roughly put, this is due to the fact that (after an 
expansion of $\ln \left(e^{x} e^{y}\right)$ in its Mercator series), the terms on any of the two sides of (6) are obtained from the terms on the other side by reordering and associating, which are not harmless procedures even in the case of real-valued series.

Another observable issue of (6) lies in the logarithm, in that, while it is uniquely given by (3) in $\mathbb{K}\langle\langle x, y\rangle\rangle$, in special Banach algebras (3) may not be the optimal choice: consider, for instance, the case of matrix algebras, where a more efficient ln-function can be defined, for many classes of matrices, through the Jordan decomposition. Since we consider the general case of Banach algebras, we are compelled to unambiguously choose what we mean by the logarithm, which we now do.

In view of the fact that the $\mathrm{BCH}$ coefficients $Z_{n}$ are constructed via the Mercator series (3) (as it is also visible from the factors $(-1)^{k+1} / k$ in (2)), it appears that (3) is the most natural choice if one wants to give a sufficiently comprehensive analysis of our problem, applicable to Banach algebras. Furthermore, (3) makes unambiguous sense in any Banach algebra $\mathcal{A}$, if we mean by $I$ the identity element of $\mathcal{A}$. For this reason, as is frequently done for operator algebras, here and in the sequel we adopt the following definition.

Definition 2.1. Let $\mathcal{A}$ be a Banach algebra, i.e., a triple $(\mathcal{A}, *,\|\cdot\|)$ where $(\mathcal{A}, *)$ is a unital associative algebra (with identity denoted by $I$ ), and $(\mathcal{A},\|\cdot\|)$ is a (real or complex) Banach space, where the norm $\|\cdot\|$ is compatible with the multiplication, i.e., $\|x * y\| \leq\|x\|\|y\|$ for any $x, y \in \mathcal{A}$.

Given $W \in \mathcal{A}, \ln (W)$ will denote the sum of the Mercator series in (31), when this series converges in the metric space $\mathcal{A}$. The function exp : $\mathcal{A} \rightarrow \mathcal{A}$ is defined via the usual series $\sum_{k=1}^{\infty} W^{k} / k$ ! (and denoted indifferently by $\exp (W)$ or $e^{W}$ ), this series being absolutely 5 convergent for any $W \in \mathcal{A}$. In what follows, given $W \in \mathcal{A}$, we say that $V \in \mathcal{A}$ is a logarithm of $W$ if $e^{V}=W$.

Finally, given any $x, y \in \mathcal{A}$, the notations in (4) will be applied for the Mercator series $\sum_{n} L_{n}(x, y)$ (be it convergent or not) and for its sum $L(x, y)$, occasionally also denoted by $\ln \left(e^{x} e^{y}\right)$.

With these definitions at hand, identity (6) is the equality of the sums of the Mercator and $\mathrm{BCH}$ series, a fact which may easily fail to be true; luckily, some positive results are available for identity (6) to hold, as the following result ensures, belonging to the $\mathrm{BCH}$ folklore 6

Proposition 2.2. Let $\mathcal{A}$ be a Banach algebra, and let $x, y \in \mathcal{A}$. Then:

(a) If $\|x\|+\|y\|<\ln 2$, the Mercator series $\sum_{n} L_{n}(x, y)$ and the $B C H$ series $\sum_{n} Z_{n}(x, y)$ are both absolutely convergent; moreover, the sums of their series are equal, and (6) holds true $\left(\ln \left(e^{x} e^{y}\right)\right.$ meaning the sum of the Mercator series).

(b) If the Mercator series $\sum_{n} L_{n}(x, y)$ converges in $\mathcal{A}$ (without any knowledge on its absolute convergence), then its sum $L(x, y)$ is a logarithm of $e^{x} e^{y}$.

(c) The same statement as in (b) is valid for the BCH series.

Problems for (6) soon arise for non-small $\|x\|+\|y\|$, as shown in the next example.

Example 2.3. Let $\mathcal{M}=M_{2}(\mathbb{R})$ denote the usual normed algebra of the real $2 \times 2$

\footnotetext{
${ }^{5}$ We say that a series $\sum_{n} a_{n}$ in $\mathcal{A}$ is absolutely convergent if $\sum_{n}\left\|a_{n}\right\|<\infty$.

${ }^{6}$ For the sake of completeness, the proof of Proposition 2.2 (based on some re-arranging argument on absolutely convergent series, and on analytic-function theory in Banach algebras) is sketched in the Appendix, as it is not so easy to locate in the literature.
} 
matrices, and let us consider (for $v \in \mathbb{R}$ ) the matrices

$$
x=x(v):=\left(\begin{array}{cc}
-v & 0 \\
0 & -2 v
\end{array}\right) \quad \text { and } \quad y:=\left(\begin{array}{cc}
0 & 1 \\
0 & 0
\end{array}\right) .
$$

In Section 3 we shall prove that:

(i) the Mercator series for $\ln \left(e^{x(v)} e^{y}\right)$ converges in $\mathcal{M}$ if and only if $v \geq-\ln \sqrt{2}$;

(ii) the $\mathrm{BCH}$ series $\sum_{n} Z_{n}(x(v), y)$ converges in $\mathcal{M}$ if and only if $|v|<2 \pi$;

(iii) there exists a logarithm of $e^{x(v)} e^{y}$ for every $v \in \mathbb{R}$; this is given e.g., by

$$
Z(v):=\left(\begin{array}{cc}
-v & \psi(v) \\
0 & -2 v
\end{array}\right)
$$

where $\psi(v):=\frac{v}{1-e^{-v}}$ is Todd's function (it is understood that $\psi(0)=1$ ).

Keeping in mind Proposition 2.2 and Example 2.3 in Section 3 we shall prove the following result, investigating in general Banach algebras problems (I)-to-(IV) previously considered for the formal-power-series algebra $\mathbb{K}\langle\langle x, y\rangle\rangle$ : we provide the mutual relationships of (I)-to-(IV), and we point out the involved pathologies for (6) to hold:

Proposition 2.4. Given $x, y$ in a Banach algebra $\mathcal{A}$, consider the problems:

(I) the Mercator series $\ln \left(e^{x} e^{y}\right)=\sum_{n} L_{n}(x, y)$ is convergent in $\mathcal{A}$;

(II) the $B C H$ series $\sum_{n}^{\infty} Z_{n}(x, y)$ is convergent in $\mathcal{A}$;

(III) there exists a logarithm of $e^{x} e^{y}$, i.e., $V \in \mathcal{A}$ fulfilling the identity $e^{V}=e^{x} e^{y}$;

(IV) the sums of the Mercator and $B C H$ series are equal.

Then the following facts hold true:

1. (II) is sufficient to (III), but not necessary.

2. (I) is sufficient to (III), but not necessary.

3. (I) and (II) are independent of each other.

4. (I), (II), (III) may all be false.

5. (I) and (II) may hold true, but (IV) can be false.

The non-convergence result of the $\mathrm{BCH}$ series contained in Example 2.3-(ii) is closely related to some recent classes of Lie algebras of interest in physics (see [25, 43]). In [43], Van-Brunt and Visser consider the case of two operators $X$ and $Y$ with the commutator relation (with scalar $u, v, c$ )

$$
[X, Y]=u X+v Y+c I
$$

where $I$ commutes with both $X$ and $Y$. When $u=v=0$, this comprises the Heisenberg case $[P, Q]=-i \hbar I$ and the creation-annihilation commutator $\left[a, a^{\dagger}\right]=I$. We observe that our Example 2.3 falls in this class: indeed, if $X$ and $Y$ are respectively given by the matrices $x$ and $y$ in (7), then (18) holds true with $u=c=0$ (and any $v$ ).

Via some formal and tricky manipulation of the $\mathrm{BCH}$ series based on a (formal) integral representation for its sum (due to Richtmyer and Greenspan, [37]), in [43] it is shown that this integral representation, under the assumption (8), is equal to

$$
X+Y+f(u, v)[X, Y], \quad \text { where } f(u, v)=\frac{u e^{u}\left(e^{v}-1\right)-v e^{v}\left(e^{u}-1\right)}{u v\left(e^{u}-e^{v}\right)}
$$


As the derivation of this object results from the $\mathrm{BCH}$ series, it seems to lead to a closed formula for the $B C H$ series, as they are usually referred to in the physics literature. Unfortunately, in general $X+Y+f(u, v)[X, Y]$ cannot be claimed to be the sum of the $\mathrm{BCH}$ series: indeed, there are suitable choices of $u, v, c$ and of $X, Y$ which give sense to $X+Y+f(u, v)[X, Y]$ but for which the $\mathrm{BCH}$ series is non-convergent: namely, take $u=c=0,|v| \geq 2 \pi$ and $X=x(v), Y=y$ in Example 2.3.

Moreover, even the Mercator logarithm $\ln \left(e^{X} e^{Y}\right)$ may be different from $X+Y+$ $f(u, v)[X, Y]$. Indeed, if we take $u=c=0, v \leq-\ln \sqrt{2}, X=x(v)$ and $Y=y$ in Example 2.3, then the Mercator series for $\ln \left(e^{X} e^{Y}\right)$ is not convergent, whereas $X+Y+f(0, v)[X, Y]$ is perfectly meaningful.

As a consequence, the following identities contained in [43]

$$
Z(X, Y)=\ln \left(e^{X} e^{Y}\right)=X+Y+f(u, v)[X, Y]
$$

must be read, in terms of the BCH series and of the Mercator logarithm, as follows: the far right-hand side, say $V$, of (9) is a prolongation (for the values of $u, v$ in the domain of $f$ ) both of the BCH series $Z(X, Y)$ and of the Mercator series for $\ln \left(e^{X} e^{Y}\right)$ when these series are not convergent; moreover, $V$ is a logarithm of $e^{X} e^{Y}$, i.e.,

$$
\exp (X+Y+f(u, v)[X, Y])=e^{X} e^{Y} .
$$

However, the existence of a prolongation of $Z(X, Y)$ does not imply the convergence of $Z(X, Y)$ and, viceversa, if a prolongation of the $\mathrm{BCH}$ series is singular at $\left(X_{0}, Y_{0}\right)$ this does not imply that $Z\left(X_{0}, Y_{0}\right)$ is non-convergent (see Section 4).

In order to clarify the possible non-convergence of the $\mathrm{BCH}$ series repeatedly mentioned above, we now state the following result, proved in Section 3 .

Theorem 2.5 (A non-convergence result for the $\mathrm{BCH}$ series). Let $\mathcal{A}$ be a Banach algebra (or, more generally, a Banach-Lie algebra) over $\mathbb{R}$ or $\mathbb{C}$. Assume that there exist $X, Y \in \mathcal{A}($ with $Y \neq 0)$ and a scalar $v$ such that $[X, Y]=v Y$.

Then the BCH series $(X+Y)+\sum_{n=2}^{\infty} Z_{n}(X, Y)$ coincides with the series

$$
(X+Y)+\sum_{n=1}^{\infty} \frac{(-v)^{n} B_{n}}{n !} Y,
$$

where the $B_{n}$ 's are the Bernoulli numbers, i.e., the rational numbers uniquely determined by the generating function $\frac{z}{e^{z}-1}=\sum_{n=0}^{\infty} \frac{B_{n}}{n !} z^{n}$. Thus the $B C H$ series $\sum_{n=1}^{\infty} Z_{n}(X, Y)$ converges in $\mathcal{A}$ if and only if $|v|<2 \pi$, and in this case the sum of the series is

$$
X+\psi(v) Y, \quad \text { where } \psi(v):=\frac{v}{1-e^{-v}} \text { is Todd's function. }
$$

In particular, if $|v| \geq 2 \pi$ the $B C H$ series $\sum_{n=1}^{\infty} Z_{n}(X, Y)$ is not convergent.

The case $Y=0$ in Theorem 2.5 is trivial, since $Z(X, 0)=X$ is always convergent.

It may be of interest to observe that, in proving Theorem [2.5, we shall not make use of the integral representation of the $Z_{n}$ 's by Richtmyer and Greenspan, [37] (as is done in [43]), but only of a simple Lie-algebra argument. 
Once it is clear that the prolongations of the maps $(X, Y) \mapsto Z(X, Y), L(X, Y)$ must not be confused with the convergence of the associated series, we think it is worthwhile to have some counterexamples at hand, showing the total independence of prolongation and convergence: these are provided in Section 4, where we shall prove the next result.

Example 2.6. Let $\mathcal{M}=M_{2}(\mathbb{C})$ denote the usual normed algebra of the complex $2 \times 2$ matrices. In $\mathcal{M}$ we consider the matrices

$$
X(\alpha):=\left(\begin{array}{cc}
-\alpha & 0 \\
0 & -2 \alpha
\end{array}\right) \text { and } Y(\beta):=\left(\begin{array}{cc}
0 & \beta(\beta-2 \pi i) \\
0 & 0
\end{array}\right) .
$$

Then the $\mathrm{BCH}$ series $Z(\alpha, \beta):=Z(X(\alpha), Y(\beta))$ converges if and only if $(\alpha, \beta)$ is in

$$
D:=\left\{(\alpha, \beta) \in \mathbb{C}^{2}:|\alpha|<2 \pi, \beta \notin\{0,2 \pi i\}\right\} \cup(\mathbb{C} \times\{0,2 \pi i\}),
$$

and the sum is given by

$$
Z(\alpha, \beta)= \begin{cases}\left(\begin{array}{cc}
-\alpha & \frac{\alpha \beta(\beta-2 \pi i)}{1-e^{-\alpha}} \\
0 & -2 \alpha
\end{array}\right), & \text { if }|\alpha|<2 \pi \text { and } \beta \notin\{0,2 \pi i\} \\
\left(\begin{array}{cc}
-\alpha & 0 \\
0 & -2 \alpha
\end{array}\right), & \text { if } \alpha \in \mathbb{C} \text { and } \beta \in\{0,2 \pi i\} .\end{cases}
$$

The interior of $D$ is $\operatorname{Int}(D)=\left\{(\alpha, \beta) \in \mathbb{C}^{2}:|\alpha|<2 \pi\right\}$, and $(\alpha, \beta) \mapsto Z(\alpha, \beta)$ is analytic here. On the other hand, the restriction of $Z(\alpha, \beta)$ to $\operatorname{Int}(D)$ can be prolonged to the $\mathcal{M}$-valued map

$$
(\alpha, \beta) \mapsto P(\alpha, \beta):=\left(\begin{array}{cc}
-\alpha & \frac{\alpha \beta(\beta-2 \pi i)}{1-e^{-\alpha}} \\
0 & -2 \alpha
\end{array}\right),
$$

which is analytic on the open set

$$
\Omega=\left\{(\alpha, \beta) \in \mathbb{C}^{2}: \alpha \neq 2 k \pi i \text { with } k \in \mathbb{Z} \backslash\{0\}\right\},
$$

and $\Omega$ clearly contains $\operatorname{Int}(D)$. Hence, the prolongation is singular at $(2 \pi i, 2 \pi i)$, whereas the $\mathrm{BCH}$ series $Z(2 \pi i, 2 \pi i)$ converges to $X(2 \pi i)$, because $(2 \pi i, 2 \pi i) \in D$.

Remark 1. The above Example 2.6 is connected with some results in [7], where the non-convergence of the $\mathrm{BCH}$ series is related to the singularity of its prolongation. Our example shows that non-convergence cannot be directly inferred from the singularity of a prolongation; Example 1 in [7] contains computations leading to the non-convergence of the $\mathrm{BCH}$ series at some singular points of its prolongation. In this sense, [7, Example 1] provides a non-trivial example where the non-convergence of the $\mathrm{BCH}$ series occurs at points where the singularity of its prolongation takes place, a phenomenon which not always happens (as Example 2.6 demonstrates).

Dual to the phenomenon depicted in Example 2.6. we have the following scenario, where the $\mathrm{BCH}$ series $Z(X, Y)$ admits a global prolongation on $\mathfrak{g} \times \mathfrak{g}$ (for a suitable real and finite-dimensional Lie algebra $\mathfrak{g})$, but the series is not everywhere convergent. 
Example 2.7. In the real algebra $M_{3}(\mathbb{R})$ of the $3 \times 3$ matrices, consider

$$
A=\left(\begin{array}{ccc}
0 & 0 & 0 \\
0 & 2 \pi & 0 \\
0 & 0 & 1
\end{array}\right), \quad B=\left(\begin{array}{ccc}
0 & 0 & 0 \\
-2 \pi & 0 & 0 \\
0 & 0 & 0
\end{array}\right), \quad C=\left(\begin{array}{ccc}
0 & 0 & 0 \\
0 & 0 & 0 \\
-1 & 0 & 0
\end{array}\right)
$$

Their commutator relations are

$$
[A, B]=2 \pi B, \quad[A, C]=C, \quad[B, C]=0 .
$$

Hence $\mathfrak{g}:=\operatorname{span}\{A, B, C\}$ is a Lie subalgebra of $M_{3}(\mathbb{R})$. By Proposition 2.2 (a), the $\mathrm{BCH}$ series $Z(X, Y)$ converges in $\mathfrak{g}$ for $X, Y \in \mathfrak{g}$ close enough to the null matrix.

In Section 4 , we shall prove that the map $(X, Y) \mapsto Z(X, Y)$ admits an analytic prolongation to the whole of $\mathfrak{g} \times \mathfrak{g}$, by using a notable abstract result by Eggert, [20]. On the other hand, since $[A, B]=2 \pi B$, we are entitled to apply Theorem 2.5] and derive that the $\mathrm{BCH}$ series $Z(A, B)$ does not converge, despite its global prolongability.

\section{A non-convergence result for the $\mathrm{BCH}$ series}

In this section we prove Theorem 2.5, the results in Example 2.3 and Proposition 2.4.

Proof (of Theorem 2.5). Taking for granted the notation in the statement of the theorem, we explicitly compute the $Z_{n}(X, Y)$ 's.

Since we know that $Z_{1}(X, Y)=X+Y$, we can suppose $n \geq 2$. With reference to the notation in Dynkin's presentation (2), from $[X, Y]=v Y,[Y, X]=-v Y$ and the trivial fact $[Y, Y]=0$, one gets that the only possibly non-vanishing summands of (2) are related to the indices for which $j_{1}+\cdots+j_{k}=1$. Thus, when $n \geq 2, Z_{n}(X, Y)$ coincides with the sum of the terms in formula (2) where $Y$ appears precisely once.

At the formal-power-series level of $\mathbb{Q}\langle\langle x, y\rangle\rangle$, we know from very classical results (see e.g., [11, eq. (4.173)]) that the sum of the terms in $\sum_{n \geq 1} Z_{n}(x, y)$ where $y$ appears exactly once is equal to

$$
\sum_{k=0}^{\infty} \frac{(-1)^{k} B_{k}}{k !}(\operatorname{ad} x)^{k}(y)
$$

where the $B_{k}$ 's are the Bernoulli numbers. Gathering these things, by degree reasons,

$$
Z_{n+1}(X, Y)=\frac{(-1)^{n} B_{n}}{n !}(\operatorname{ad} X)^{n}(Y), \quad \forall n \geq 1 .
$$

On the other hand, from $[X, Y]=v Y$ one gets $(\operatorname{ad} X)^{n}(Y)=v^{n} Y$ for $n \geq 1$, so that

$$
Z_{n+1}(X, Y)=\frac{(-1)^{n} B_{n}}{n !} v^{n} Y, \quad \forall n \geq 1 .
$$

Thus, the BCH series $\sum_{n=1}^{\infty} Z_{n}(X, Y)=(X+Y)+\sum_{n=2}^{\infty} Z_{n}(X, Y)$ coincides with

$$
(X+Y)+\sum_{n=1}^{\infty} \frac{(-1)^{n} B_{n}}{n !} v^{n} Y=X+\left(\sum_{n=0}^{\infty} \frac{B_{n}}{n !}(-v)^{n}\right) Y
$$


Since the $B_{n}$ 's are defined by $\frac{z}{e^{z}-1}=\sum_{n=0}^{\infty} \frac{B_{n}}{n !} z^{n}$, the radius of convergence of the power series $\sum_{n=0}^{\infty} \frac{B_{n}}{n !} z^{n}$ is $2 \pi$. It can be proved that the power series does not converge when $|z|=2 \pi$ (for completeness reason, we furnish the proof of this in Remark 2).

This shows that (since $Y \neq 0$ ) the $\mathrm{BCH}$ series $\sum_{n=1}^{\infty} Z_{n}(X, Y)$ converges if and only if $|v|<2 \pi$. As for its sum, if $|v|<2 \pi$ we have

$$
\sum_{n=0}^{\infty} \frac{B_{n}}{n !}(-v)^{n}=\frac{-v}{e^{-v}-1}=\psi(v),
$$

so that, on account of (15), we get (10). This ends the proof of Theorem 2.5,

Next we prove the results in Example 2.3. For $v \in \mathbb{R}$, let $X=X(v)$ and $Y$ be respectively the matrices $x=x(v)$ and $y$ in (7). A direct computation shows that

$$
[X, Y]=\left(\begin{array}{ll}
0 & v \\
0 & 0
\end{array}\right)=v Y
$$

so that (with $\mathcal{A}=M_{2}(\mathbb{R})$ ) we are entitled to apply Theorem 2.5. Hence assertion (ii) of Example 2.3 follows directly from that theorem. By a direct computation we have

$$
e^{X} e^{Y}=\left(\begin{array}{cc}
e^{-v} & 0 \\
0 & e^{-2 v}
\end{array}\right) \cdot\left(\begin{array}{cc}
1 & 1 \\
0 & 1
\end{array}\right)=\left(\begin{array}{cc}
e^{-v} & e^{-v} \\
0 & e^{-2 v}
\end{array}\right)
$$

Thus, the Mercator series (4) boils down to the matrix series (be it convergent or not)

$$
\sum_{n=1}^{\infty} \frac{(-1)^{n+1}}{n}\left(\begin{array}{cc}
e^{-v}-1 & e^{-v} \\
0 & e^{-2 v}-1
\end{array}\right)^{n} .
$$

When $v=0$ this series trivially converges, and its sum is equal to $Y$; hence we can assume $v \neq 0$. By a direct diagonalization, we have

$$
\left(\begin{array}{cc}
e^{-v}-1 & e^{-v} \\
0 & e^{-2 v}-1
\end{array}\right)=\left(\begin{array}{cc}
1 & 1 \\
0 & e^{-v}-1
\end{array}\right) \cdot\left(\begin{array}{cc}
e^{-v}-1 & 0 \\
0 & e^{-2 v}-1
\end{array}\right) \cdot\left(\begin{array}{cc}
1 & \frac{1}{1-e^{-v}} \\
0 & \frac{-1}{1-e^{-v}}
\end{array}\right) .
$$

As a consequence, the series (16) is equal to

$$
\left(\begin{array}{cc}
1 & 1 \\
0 & e^{-v}-1
\end{array}\right) \cdot\left(\begin{array}{cc}
\sum_{n=1}^{\infty} \frac{(-1)^{n+1}}{n}\left(e^{-v}-1\right)^{n} & 0 \\
0 & \sum_{n=1}^{\infty} \frac{(-1)^{n+1}}{n}\left(e^{-2 v}-1\right)^{n}
\end{array}\right) \cdot\left(\begin{array}{cc}
1 & \frac{1}{1-e^{-v}} \\
0 & \frac{-1}{1-e^{-v}}
\end{array}\right) .
$$

Now this series is convergent if and only if $e^{-v}-1$ and $e^{-2 v}-1$ belong to ] $\left.-1,1\right]$, and this is equivalent to $v \geq-\ln \sqrt{2}$. This proves assertion (i) of Example 2.3. Finally, assertion (iii), which is equivalent to

$$
\exp \left(\begin{array}{cc}
-v & \frac{v}{1-e^{-v}} \\
0 & -2 v
\end{array}\right)=\left(\begin{array}{cc}
e^{-v} & e^{-v} \\
0 & e^{-2 v}
\end{array}\right) \quad \forall v \in \mathbb{R}
$$

can be proved by a direct diagonalization. Finally, we provide the following 
Proof (of Proposition 2.4). Numbers are related to the statements in the thesis of Proposition 2.4

(1) Sufficiency follows from statement (c) in Proposition 2.2, The lack of necessity is shown in Example 2.3, if one takes $|v| \geq 2 \pi$.

(2) Sufficiency follows from statement (b) in Proposition 2.2. The lack of necessity is shown in Example 2.3, if one takes $v<-\ln \sqrt{2}$. Another simpler example: if one chooses $\mathcal{A}=\mathbb{R}, y=0$ and $x>\ln 2$, then (III) holds with $V=x$, but $\sum_{n=1}^{\infty}(-1)^{n+1}\left(e^{x}-1\right)^{n} / n$ is not convergent.

(3) On the one hand, it is simple to show that (II) may hold without (I): for instance, in $\mathbb{R}$, if we take $x>\ln 2$ and $y=0$, then the BCH series boils down to $\sum_{n=1}^{\infty} Z_{n}(x, 0)=x$ (and is therefore trivially convergent), whereas the Mercator series for $\ln \left(e^{x} e^{y}\right)$ does not converge, as observed above. A less trivial example is again given by Example 2.3, by taking $v \in(-2 \pi,-\ln \sqrt{2})$. Vice versa, the choice $v>2 \pi$ yields an example for which (I) holds true but (II) is false.

(4) Taking the example by Wei [47] related to the Banach algebra $\mathcal{M}=M_{2}(\mathbb{R})$ and the matrices

$$
W:=\left(\begin{array}{cc}
0 & -5 \pi / 4 \\
5 \pi / 4 & 0
\end{array}\right) \text { and } Y:=\left(\begin{array}{ll}
0 & 1 \\
0 & 0
\end{array}\right)
$$

it can be proved that there does not exist any logarithm of $e^{W} e^{Y}$ in $\mathcal{M}$. Thus, in view of statements (b) and (c) in Proposition 2.2. both the $\mathrm{BCH}$ series $\sum_{n} Z_{n}(W, Y)$ and the Mercator series $\sum_{n} L_{n}(W, Y)$ cannot converge, otherwise they would provide such a logarithm.

(5) It can be easily seen that, with the following choice

$$
A:=\left(\begin{array}{cc}
\ln 2 & -2 \pi \\
2 \pi & \ln 2
\end{array}\right) \quad \text { and } \quad B:=\left(\begin{array}{ll}
0 & 0 \\
0 & 0
\end{array}\right)
$$

then (as $A, B$ commute) the $\mathrm{BCH}$ series boils down to $Z(A, B)=Z_{1}(A, B)=$ $A+B=A$, whereas the Mercator series is given by

$$
L(A, B)=\sum_{n=1}^{\infty} \frac{(-1)^{n+1}}{n}\left(\begin{array}{ll}
1 & 0 \\
0 & 1
\end{array}\right)^{n}=\left(\begin{array}{cc}
\ln 2 & 0 \\
0 & \ln 2
\end{array}\right) .
$$

Thus, $Z(A, B) \neq L(A, B)$ even if they are both convergent.

This ends the proof.

\section{Prolongation issues for the $\mathrm{BCH}$ series}

As a first prolongation problem, we prove the assertions in Example 2.6, showing that the $\mathrm{BCH}$ series $Z(X, Y)$ can possess an analytic prolongation which is singular at $\left(X_{0}, Y_{0}\right)$, whereas $Z\left(X_{0}, Y_{0}\right)$ converges.

Proof (of Example 2.6). Let $X(\alpha), Y(\beta) \in \mathcal{M}=M_{2}(\mathbb{C})$ be as in (11). Note that

$$
[X(\alpha), Y(\beta)]=\alpha Y(\beta)
$$


When $\beta \in\{0,2 \pi i\}$, we have $Y(\beta)=0$ and the $\mathrm{BCH}$ series $Z(\alpha, \beta):=Z(X(\alpha), Y(\beta))$ boils down to $X(\alpha)$. Instead, when $\beta \notin\{0,2 \pi i\}$, we can apply Theorem 2.5, deducing the convergence of $Z(\alpha, \beta)$ precisely when $|\alpha|<2 \pi$ to the sum (see (10))

$$
X(\alpha)+\psi(\alpha) Y(\beta)=\left(\begin{array}{cc}
-\alpha & \frac{\alpha \beta(\beta-2 \pi i)}{1-e^{-\alpha}} \\
0 & -2 \alpha
\end{array}\right)
$$

Gathering these facts, we obtain that $Z(\alpha, \beta)$ converges precisely on the set $D$ defined in (12) and its sum is (13). Since $\operatorname{Int}(D)=\left\{(\alpha, \beta) \in \mathbb{C}^{2}:|\alpha|<2 \pi\right\}$, and since

$$
(\alpha, \beta) \mapsto P(\alpha, \beta):=\left(\begin{array}{cc}
-\alpha & \frac{\alpha \beta(\beta-2 \pi i)}{1-e^{-\alpha}} \\
0 & -2 \alpha
\end{array}\right)
$$

is naturally defined and analytic on the open set

$$
\Omega=\left\{(\alpha, \beta) \in \mathbb{C}^{2}: \alpha \neq 2 k \pi i \text { with } k \in \mathbb{Z} \backslash\{0\}\right\},
$$

we see by direct inspection that the restriction of $Z(\alpha, \beta)$ to $\operatorname{Int}(D)$ can be analytically prolonged by the function $P(\alpha, \beta)$ on $\Omega \supset \operatorname{Int}(D)$.

We note that $P$ is singular at $(2 \pi i, 2 \pi i)$, since one has

$$
P\left(2 \pi i+\varepsilon^{2}, 2 \pi i+\varepsilon\right)=\left(\begin{array}{cc}
-2 \pi i-\varepsilon^{2} & \frac{\left(2 \pi i+\varepsilon^{2}\right)(2 \pi i+\varepsilon) \varepsilon}{1-e^{-\varepsilon^{2}}} \\
0 & -4 \pi i-2 \varepsilon^{2}
\end{array}\right)
$$

and the limit of the $(1,2)$-entry as $\varepsilon \rightarrow 0$ does not exist. However, the $\mathrm{BCH}$ series $Z(2 \pi i, 2 \pi i)$ converges since $(2 \pi i, 2 \pi i) \in D$.

We are left to prove what is stated in Example 2.7, showing a prolongation issue dual to that highlighted in Example 2.6. we provide a Lie algebra of real matrices in which the $\mathrm{BCH}$ series is not everywhere convergent, yet admitting a global prolongation.

Proof (of Example 2.7). Let $A, B, C$ be as in (14) and let $\mathfrak{g}=\operatorname{span}\{A, B, C\}$. In order to prove the existence of a global extension of the $\mathrm{BCH}$ series $Z(X, Y)$ to the whole of $\mathfrak{g} \times \mathfrak{g}$, we apply a general abstract result by Eggert, [20].

Indeed, Theorem 4.4 in [20] proves that the $\mathrm{BCH}$ operation $(X, Y) \mapsto Z(X, Y)$ (certainly well posed when $X, Y$ are close to $0 \in \mathfrak{g}$ ) can be analytically continued to the whole of $\mathfrak{g} \times \mathfrak{g}$ if and only if the connected and simply connected Lie group $\mathbb{G}$ associated with $\mathfrak{g}$ by Lie's Third Theorem is globally isomorphic to $\mathfrak{g}$ via the exponential map.

Now, by some computations in [12], we know that the following Lie group does exactly this job: $\mathbb{G}=\left(\mathbb{R}^{3}, \cdot\right)$ where

$$
x \cdot y=\left(x_{1}+y_{1}, x_{2}+e^{2 \pi x_{1}} y_{2}, x_{3}+e^{x_{1}} y_{3}\right) .
$$

Indeed, the Lie algebra Lie $(\mathbb{G})$ of $\mathbb{G}$ is the Lie algebra of vector fields spanned by

$$
X_{1}:=\frac{\partial}{\partial x_{1}}, \quad X_{2}:=e^{2 \pi x_{1}} \frac{\partial}{\partial x_{2}}, \quad X_{3}:=e^{x_{1}} \frac{\partial}{\partial x_{3}}
$$


and the linear map sending $X_{1}, X_{2}, X_{3}$ to $A, B, C$ (respectively) is an isomorphism of Lie algebras between $\operatorname{Lie}(\mathbb{G})$ and $\mathfrak{g}$.

After some tedious computations (see [12]), one recognizes that the exponential map $\operatorname{Exp}: \operatorname{Lie}(\mathbb{G}) \rightarrow \mathbb{G}$ is explicitly given by

$$
\operatorname{Exp}\left(\xi_{1} X_{1}+\xi_{2} X_{2}+\xi_{3} X_{3}\right)=\left(\xi_{1}, \xi_{2} \frac{e^{2 \pi \xi_{1}}-1}{2 \pi \xi_{1}}, \xi_{3} \frac{e^{\xi_{1}}-1}{\xi_{1}}\right), \quad \xi_{1}, \xi_{2}, \xi_{3} \in \mathbb{R}
$$

which is clearly invertible, with smooth inverse map.

Summing up, by [20, Theorem 4.4] we infer that the map $(X, Y) \mapsto Z(X, Y)$ can be analytically continued to the whole of $\mathfrak{g} \times \mathfrak{g}$. However, $Z(A, B)$ does not converge, as it follows by our Theorem 2.5, since $[A, B]=2 \pi B$.

Finally, somewhat connected with [7, Theorem 4.1] (related to an unpublished result by Mityagin) on the improved convergence domain $\{\|X\|+\|Y\|<\pi\}$ for the $\mathrm{BCH}$ series $Z(X, Y)$ in matrix Lie algebras, we give the next example; this shows that the the latter improved domain is not a convergence domain for the Mercator logarithm $L(X, Y)$.

Example 4.1. Consider the matrices $x(v)$ and $y$ in (7), for $v \in \mathbb{R}$. By Example 2.3 we already know that the Mercator series $L(x(v), y)$ for $\ln \left(e^{x(v)} e^{y}\right)$ converges in the Banach algebra $\mathcal{M}$ of the $2 \times 2$ real matrices if and only if $v \geq-\ln \sqrt{2}$.

As a consequence, if we take $v=-1$, the Mercator series $L(x(-1), y)$ does not converge, even if $\|x(-1)\|+\|y\|=3<\pi$. Here we are considering the operator norm

$$
\|A\|=\sup _{|x|=1}|A x|, \quad A \in \mathcal{M},
$$

and $|\cdot|$ is the standard Euclidean norm on $\mathbb{R}^{2}$.

\section{Appendix}

For the sake of completeness, we give the following:

Proof of Proposition 2.2. We split the proof according to the statement of the proposition.

(a). Let $\|x\|+\|y\|<\ln 2$. Then, by the compatibility of the norm of $\mathcal{A}$ with the product, we have (see (4))

$$
\begin{aligned}
\sum_{n=1}^{\infty}\left\|L_{n}(x, y)\right\| & \leq \sum_{n=1}^{\infty} \frac{1}{n} \sum_{\left(i_{1}, j_{1}\right), \ldots,\left(i_{n}, j_{n}\right) \neq(0,0)} \frac{\|x\|^{i_{1}}\|y\|^{j_{1}} \cdots\|x\|^{i_{n}}\|y\|^{j_{n}}}{i_{1} ! j_{1} ! \cdots i_{n} ! j_{n} !} \\
& =\sum_{n=1}^{\infty} \frac{1}{n}\left(e^{\|x\|+\|y\|}-1\right)^{n}=-\ln \left(2-e^{\|x\|+\|y\|}\right)<\infty .
\end{aligned}
$$

The last equality is a consequence of $\|x\|+\|y\|<\ln 2$ and the trivial fact $\sum_{n} w^{n} / n=$ $-\ln (1-w)$, valid for $w \in[-1,1)$. This proves the absolute convergence of the Mercator series $L(x, y)$ when $\|x\|+\|y\|<\ln 2$. The above computation shows that we can rearrange the sums over $n$ and over $\left(i_{1}, j_{1}\right), \ldots,\left(i_{n}, j_{n}\right)$ as we please. We group 
homogeneous terms of the same degree as follows:

$$
\begin{aligned}
& L_{n}(x, y)=\sum_{k=n}^{\infty} L_{n, k}(x, y) \quad \text { where } \\
& L_{n, k}(x, y):=\frac{(-1)^{n+1}}{n} \sum_{\substack{\left(i_{1}, j_{1}\right), \ldots,\left(i_{n}, j_{n}\right) \neq(0,0) \\
i_{1}+j_{1}+\cdots+i_{n}+j_{n}=k}} \frac{x^{i_{1}} y^{j_{1}} \cdots x^{i_{n}} y^{j_{n}}}{i_{1} ! j_{1} ! \cdots i_{n} ! j_{n} !} .
\end{aligned}
$$

This gives the computation (the sums can be interchanged due to absolute convergence)

$$
L(x, y)=\sum_{n=1}^{\infty} L_{n}(x, y)=\sum_{n=1}^{\infty} \sum_{k=n}^{\infty} L_{n, k}(x, y)=\sum_{k=1}^{\infty} \sum_{n=1}^{k} L_{n, k}(x, y) .
$$

We claim that the last member is equal to the $\mathrm{BCH}$ series $\sum_{k=1}^{\infty} Z_{k}(x, y)$. This will give the equality $L(x, y)=Z(x, y)$ when $\|x\|+\|y\|<\ln 2$. The claim is a consequence of the following fact:

$$
\sum_{n=1}^{k} L_{n, k}(x, y)=\sum_{n=1}^{k} \frac{(-1)^{n+1}}{n} \sum_{\substack{\left(i_{1}, j_{1}\right), \ldots,\left(i_{n}, j_{n}\right) \neq(0,0) \\ i_{1}+j_{1}+\cdots+i_{n}+j_{n}=k}} \frac{x^{i_{1}} y^{j_{1}} \cdots x^{i_{n}} y^{j_{n}}}{i_{1} ! j_{1} ! \cdots i_{n} ! j_{n} !}=Z_{k}(x, y) .
$$

Indeed, the last equality holds true since the middle term is precisely the associative presentation of $Z_{k}(x, y)$ in $\mathbb{K}\langle\langle x, y\rangle\rangle$ (the one leading to Dynkin's presentation (2) after an application of the Dynkin-Specht-Wever map), see e.g., [11, Sec. 3.1.3]. We are left to prove the absolute convergence of the $\mathrm{BCH}$ series when $\|x\|+\|y\|<\ln 2$ :

$$
\begin{aligned}
\sum_{k=1}^{\infty}\left\|Z_{k}(x, y)\right\| & \stackrel{(18)}{\leq} \sum_{k=1}^{\infty} \sum_{n=1}^{k} \frac{1}{n} \sum_{\substack{\left(i_{1}, j_{1}\right), \ldots,\left(i_{n}, j_{n}\right) \neq(0,0) \\
i_{1}+j_{1}+\cdots+i_{n}+j_{n}=k}} \frac{\|x\|^{i_{1}+\cdots+i_{n}}\|y\|^{j_{1}+\cdots+j_{n}}}{i_{1} ! j_{1} ! \cdots i_{n} ! j_{n} !} \\
& =\sum_{n=1}^{\infty} \sum_{k=n}^{\infty} \frac{1}{n} \sum_{\substack{\left(i_{1}, j_{1}\right), \ldots,\left(i_{n}, j_{n}\right) \neq(0,0) \\
i_{1}+j_{1}+\cdots+i_{n}+j_{n}=k}} \frac{\|x\|^{i_{1}+\cdots+i_{n}}\|y\|^{j_{1}+\cdots+j_{n}}}{i_{1} ! j_{1} ! \cdots i_{n} ! j_{n} !} \\
& =\sum_{n=1}^{\infty} \frac{1}{n} \sum_{\left(i_{1}, j_{1}\right), \ldots,\left(i_{n}, j_{n}\right) \neq(0,0)} \frac{\|x\|^{i_{1}+\cdots+i_{n}}\|y\|^{j_{1}+\cdots+j_{n}}}{i_{1} ! j_{1} ! \cdots i_{n} ! j_{n} !} \\
& \stackrel{(17)}{=}-\ln \left(2-e^{\|x\|+\|y\|}\right) .
\end{aligned}
$$

(b). Suppose that $\sum_{n} L_{n}(x, y)$ converges in $\mathcal{A}$. Then, by Abel's Lemma in Banach spaces (see e.g., [11, Lemma 5.68]), we know that the power series $F(t):=$ $\sum_{n} L_{n}(x, y) t^{n}$ is uniformly convergent (hence continuous) for $t$ in $[0,1]$, and is an $\mathcal{A}$-valued analytic function on $(0,1)$. Now it is a standard fact to show that there exists $\epsilon>0$ such that

$$
\exp \left(\sum_{n=1}^{\infty} \frac{(-1)^{n+1}}{n} w^{n}\right)=I+w, \quad \text { for every } w \in \mathcal{A} \text { such that }\|w\|<\epsilon .
$$


As a consequence, if $t$ is suitably small (so that $\left\|t\left(e^{x} e^{y}-I\right)\right\|<\epsilon$ ) we have

$$
\begin{gathered}
\exp (F(t))=\exp \left(\sum_{n=1}^{\infty} L_{n}(x, y) t^{n}\right) \stackrel{(4)}{=} \exp \left(\sum_{n=1}^{\infty} \frac{(-1)^{n+1}}{n}\left(t\left(e^{x} e^{y}-I\right)\right)^{n}\right) \\
\stackrel{(19)}{=} I+t\left(e^{x} e^{y}-I\right)=: G(t) .
\end{gathered}
$$

Thus, exp $\circ F$ and $G$ are two $\mathcal{A}$-valued analytic functions on $(0,1)$, coinciding on some small interval $\left(0, \epsilon^{\prime}\right)$, with $\epsilon^{\prime}>0$. By Unique Continuation we infer that $\exp \circ F=G$ on $(0,1)$, and by continuity we get $\exp (F(1))=G(1)$. The latter identity is precisely $\exp (L(x, y))=e^{x} e^{y}$.

(c). We set $F(t):=\sum_{n} Z_{n}(t x, t y)$. Since $Z_{n}$ is a homogeneous polynomial of degree $n$, we have $F(t)=\sum_{n} Z_{n}(x, y) t^{n}$. Arguing as above we know that the power series $F(t)$ is uniformly convergent (hence continuous) for $t$ in $[0,1]$, and an $\mathcal{A}$-valued analytic function on $(0,1)$. When $t$ is small (say $t \in[0, \epsilon]$ with $\epsilon=\epsilon(x, y)>0$ ), we have $\|t x\|+\|t y\|<\ln 2$, hence (by part (a) of the proof) $F(t)$ is a logarithm of $e^{t x} e^{t y}$ :

$$
\exp (F(t))=e^{t x} e^{t y} \quad \forall t \in[0, \epsilon] .
$$

Now, both sides of this identity are analytic functions of $t$ on $(0,1)$, hence this identity is valid throughout $(0,1)$ by Unique Continuation. By continuity, the identity remains true for $t=1$ :

$$
\exp (F(1))=e^{x} e^{y}, \quad \text { i.e., } \quad \exp \left(\sum_{n} Z_{n}(x, y)\right)=e^{x} e^{y}
$$

This is exactly what we wanted to prove.

Next, we review some special function facts for the non-convergence of the series associated with the Bernoulli numbers on the boundary of the disc of convergence.

Remark 2. Let the $B_{n}$ 's be as in Theorem 2.5. We show that the power series $S(z):=$ $\sum_{n=0}^{\infty} \frac{B_{n}}{n !} z^{n}$ is not convergent on the boundary $\{|z|=2 \pi\}$ of its convergence disc; since $B_{2 n+1}=0$ for every $n \geq 1, S(z)$ converges if and only if the series $\widetilde{S}(z):=$ $\sum_{n=1}^{\infty} \frac{B_{2 n}}{(2 n) !} z^{2 n}$ converges. We use a formula relating the $B_{n}$ 's to Riemann's $\zeta$ function (see [46, §3.16, p. 117]):

$$
\zeta(2 n)=\frac{(2 \pi)^{2 n}(-1)^{n-1} B_{2 n}}{2 \cdot(2 n) !}, \quad \text { for every } n \in \mathbb{N} .
$$

Since $\zeta(2 n) \longrightarrow 1$ as $n \rightarrow \infty$, we get $\frac{\left|B_{2 n}\right|}{(2 n) !} \sim \frac{2}{(2 \pi)^{2 n}}$ as $n \rightarrow \infty$. Therefore, if $|z|=2 \pi$, then $\widetilde{S}(z)$ cannot converge, since $\frac{\left|B_{2 n}\right|}{(2 n) !}|z|^{2 n} \longrightarrow 2$ as $n \rightarrow \infty$.

\section{Acknowledgements}

The authors would like to thank the anonymous Referee of this paper whose constructive criticism lead us to improve a former version of the manuscript. 


\section{References}

[1] R. Achilles, A. Bonfiglioli: The early proofs of the Theorem of Campbell, Baker, Hausdorff and Dynkin, Arch. Hist. Exact Sci., 66, 295-358 (2012).

[2] M. Bauer, R. Chetrite, K. Ebrahimi-Fard, F. Patras: Time-ordering and generalized Magnus expansion, Lett. Math. Phys. 103, 331-350 (2013).

[3] G. Basar, G. V. Dunne and M. Unsal: Quantum geometry of resurgent perturbative/nonperturbative relations, JHEP 1705, 087 (2017).

[4] S. Biagi, A. Bonfiglioli: On the convergence of the Campbell-Baker-Hausdorff-Dynkin series in infinite-dimensional Banach-Lie algebras, Linear Multilin. Algebra 62, 1591-1615 (2014).

[5] S. Biagi, A. Bonfiglioli: A completeness result for time-dependent vector fields and applications, Commun. Contemp. Math. 17, 1-26 (2015).

[6] S. Biagi, A. Bonfiglioli: The existence of a global fundamental solution for homogeneous Hörmander operators via a global Lifting method, Proc. London Math. Soc. 114, 855-889 (2017).

[7] S. Blanes, F. Casas: On the convergence and optimization of the Baker-Campbell-Hausdorff formula, Linear Algebra Appl. 378, 135-58 (2004).

[8] S. Blanes, F. Casas, J. A. Oteo, J. Ros: Magnus and Fer expansions for matrix differential equations: The convergence problem, J. Phys. A: Math. Gen. 22, 259-268 (1998).

[9] S. Blanes, F. Casas, J. A. Oteo, J. Ros: The Magnus expansion and some of its applications, Phys. Rep. 470, 151-238 (2009).

[10] A. Bonfiglioli: An ODE's version of the formula of Baker, Campbell, Dynkin and Hausdorff and the construction of Lie groups with prescribed Lie algebra, Mediterr. J. Math. 7, 387-414 (2010).

[11] A. Bonfiglioli, R. Fulci: Topics in Noncommutative Algebra. The Theorem of Campbell, Baker, Hausdorff and Dynkin. Lecture Notes in Mathematics, 2034, Springer-Verlag: Heidelberg, 2012. ISBN: 978-3-642-22596-3.

[12] A. Bonfiglioli, E. Lanconelli: Lie groups related to Hörmander operators and KolmogorovFokker-Planck equations, Commun. Pure Appl. Anal. 11, 1587-1614 (2012).

Lie groups related to Hörmander operators and Kolmogorov-Fokker-Planck equations. Commun. Pure Appl. Anal.2012;11(5):1587-614.

[13] N. Bourbaki: Éléments de Mathématique. Groupes et Algèbres de Lie. Chap. I-III, Actualités scientifiques et industrielles. Paris: Hermann, 1972.

[14] A. Bravetti, A. A. Garcia-Chung and D. Tapias: Exact Baker-Campbell-Hausdorff formula for the contact Heisenberg algebra, J. Phys. A 50 no.10, 105203 (2017).

[15] F. Casas: Sufficient conditions for the convergence of the Magnus expansion, J. Phys. A: Math. Theor. 40, 15001-15017 (2007).

[16] F. Casas, A. Murua: An efficient algorithm for computing the Baker-Campbell-Hausdorff series and some of its applications, J. Math. Phys. 50, 033513-1-033513-23 (2009).

[17] J. Day, W. So, W., R.C. Thompson: Some properties of the Campbell-Baker-Hausdorff series, Linear and Multilinear Algebra 29, 207-224 (1991).

[18] E. B. Dynkin: Normed Lie algebras and analytic groups, Uspehi Matem. Nauk (N.S.) 5, n.1 (35), 135-186 (1950).

[19] J. Écalle: Les fonctions resurgentes, vol. I - III Publ. Math. Orsay, 1981.

[20] A. Eggert: Extending the Campbell-Hausdorff multiplication, Geom. Dedicata 46, 35-45 (1993).

[21] D. L. Foulis: The algebra of complex $2 \times 2$ matrices and a general closed Baker-CampbellHausdorff formula, J. Phys. A 50 no.30, 305204 (2017).

[22] F. Hausdorff: Die symbolische Exponentialformel in der Gruppentheorie, Leipz. Ber. 58, 19-48 (1906).

[23] T. Kimura: Explicit description of the Zassenhaus formula, PTEP 2017 no.4, 041A03 (2017).

[24] Y. Li, D. Sauzin, S. Sun: The Baker-Campbell-Hausdorff formula via mould calculus, arXiv:1805.00766 [math.RA]. 
[25] C.F. Lo: Comment on 'Special-case closed form of the Baker-Campbell-Hausdorff formula', J. Phys. A: Math. Theor. 49, 218001 (2016).

[26] M. Matone: An algorithm for the Baker-Campbell-Hausdorff formula, JHEP 1505, 113 (2015).

[27] M. Matone: Classification of commutator algebras leading to the new type of closed BakerCampbell-Hausdorff formulas, J. Geom. Phys. 97, 34-43 (2015).

[28] M. Matone: Closed form of the Baker-Campbell-Hausdorff formula for the generators of semisimple complex Lie algebras, Eur. Phys. J. C 76 no.11, 610 (2016).

[29] M. Matone, P. Pasti: Wilson loops on Riemann surfaces, Liouville theory and covariantization of the conformal group, JHEP 1506, 010 (2015).

[30] M. Mérigot: Domaine de convergence de la série de Campbell-Hausdorff, multigraphie interne, Nice University (1974).

[31] J. Michel: Bases des algèbres de Lie et série de Hausdorff, Séminaire Dubreil. Algèbre 27 n.1 (1973-1974), exp. n.6, 1-9 (1974).

[32] P. C. Moan: Efficient approximation of Sturm-Liouville problems using Lie-group methods, Technical Report 1998/NA11, Dep. of Applied Mathematics and Theoretical Physics, University of Cambridge, England, 1998.

[33] P. C. Moan, J. Niesen: Convergence of the Magnus series, Found. Comput. Math. 8, 291$301(2008)$.

[34] P. C. Moan, J.A. Oteo: Convergence of the exponential Lie series, J. Math. Phys. 42, 501-508 (2001).

[35] M. Newman, W. So, R. C. Thompson: Convergence domains for the Campbell-BakerHausdorff formula, Linear Multilinear Algebra 24, 301-310 (1989).

[36] M. Postnikov: Lie groups and Lie algebras. Lectures in geometry. Semester V. Translated from the Russian. "Mir", Moscow, 1986.

[37] R. D. Richtmyer, S. Greenspan: Expansion of the Campbell-Baker-Hausdorff formula by computer, Comm. Pure Appl. Math. 18, 107-108 (1965).

[38] R.S. Strichartz: The Campbell-Baker-Hausdorff-Dynkin formula and solutions of differential equations, J. Funct Anal. 72, 320-345 (1987).

[39] F. Strocchi: An introduction to the mathematical structure of quantum mechanics. Advanced Series in Mathematical Physics, Vol. 28. World Scientific Publishing Co. Inc.: Singapore, 2008.

[40] M. Suzuki: On the convergence of exponential operators-the Zassenhaus formula, BCH formula and systematic approximants, Commun. Math. Phys. 57, 193-200 (1977).

[41] S. Tamura, H. Katsura: Zero-energy states in conformal field theory with sine-square deformation, PTEP 2017, 113 (2017).

[42] R. C. Thompson: Convergence proof for Goldberg's exponential series, Linear Algebra Appl. 121, 3-7 (1989).

[43] A. Van-Brunt, M. Visser: Special-case closed form of the Baker-Campbell-Hausdorff formula, J. Phys. A: Math. Theor. 48, 225207 (2015).

[44] M. Serone, G. Spada, G. Villadoro: The power of perturbation theory, JHEP 1705, 056 (2017).

[45] V.A. Vinokurov: The logarithm of the solution of a linear differential equation, Hausdorff's formula, and conservation laws, Soviet Math. Dokl., 44, 200-205 (1992). Translated from 319, no.5 (1991).

[46] Z. X. Wang, D. R. Guo: Special Functions. World Scientific Publishing Co. Inc.: Singapore, 1989.

[47] J. Wei: Note on the global validity of the Baker-Hausdorff and Magnus theorems, J. Mathematical Phys. 4, 1337-1341 (1963). 